MPP-2016-300

October 3, 2018

\title{
Studies of correlations between measurements of jet observables
}

\author{
Andrii Verbytskyila \\ aMax-Planck-Institut für Physik (Werner-Heisenberg-Institut), \\ Föringer Ring 6, München 80805, Germany
}

\begin{abstract}
We present a method for calculation of statistical correlations between measured jet observables in high energy collisions. The case of measurements of jet rates in $e^{+} e^{-}$collisions is considered in detail. The method is compared to sampling based methods used in the past.
\end{abstract}

${ }^{1}$ andrii.verbytskyi@mpp.mpg.de 


\section{Introduction}

In high energy particle collisions the partons and hadrons are produced in collimated bunches called jets. In a very simplified model a jet can be considered as a parton that was produced in a hard process and went through showering and hadronisation processes. The studies of jet production in the $e^{+} e^{-}, e^{ \pm} p$ and $p p$ collisions serve as a strong test of Quantum Chromodynamics (QCD). For instance, comparisons of the jet production cross sections in $e^{+} e^{-}$collisions to the fixed order or resummed perturbative QCD predictions are used for the extraction of the strong coupling constant $\alpha_{s}\left(M_{Z}\right)$ [1]4]. For the ultimate precision of the $\alpha_{s}\left(M_{Z}\right)$ determination, several measured jet observables are combined. In this case the statistical and systematical correlations between the measurements of jet observables are important. To estimate the statistical correlations between the measured quantities, several methods were used in the past.

In this work jet observables classes are introduced and are used to calculate other jet observables and obtain statistical correlations between them in a model-independent way using only data. To illustrate the method, calculations of jet rates in the $e^{+} e^{-}$collisions, multijet cross sections in $p p$ collisions and inclusive jet cross sections in $e^{ \pm} p$ collisions are considered in toy analyses with Monte Carlo (MC) simulated events. The results for jet rates in $e^{+} e^{-}$collisions are compared to those obtained with sampling method used in the past [2-5].

\section{Jet algorithms}

A jet clustering algorithm is a way to simplify high energy collision event topology and exhibit the underlying physics at the parton level. The main goal of such a procedure is to reconstruct the kinematic variables of the partons produced during the primary hard interaction. The energy and the momenta of the partons are reconstructed by combining momenta and energy of the charged and neutral particles which are clustered into jets. Several jet algorithms are used to perform the combination in different environments $-e^{+} e^{-}, p p$ or $e^{ \pm} p$ collisions. A detailed overview of their properties can be found elsewhere [6,7], only some properties are briefly discussed below.

The number of jets reconstructed a given collision event depends on the applied jet algorithm and used cut parameters. The former can be illustrated with the $k_{T}$ algorithm [8] widely used in $e^{+} e^{-}$jet studies. In this algorithm a measure $y_{i j}$ is defined for a pair of particles $i$ and $j$ with total visible energy in the event $E_{\mathrm{vis}}$, energies of particles $E_{i}, E_{j}$ 
and angle between them $\theta_{i j}$

$$
y_{i j}=\frac{2 \min \left(E_{i}^{2}, E_{j}^{2}\right)\left(1-\cos \theta_{i j}\right)}{E_{\mathrm{vis}}^{2}} .
$$

At every step of the iterative recombination procedure, all distances are calculated and a pair of particles with minimal distance between them is determined. As long as this distance is smaller than cut parameter $y$, these particles are combined by adding their 4 -vectors and next iteration begins. In this way $y$ defines the number of jets in the event.

To define the number of jets obtained with cone-like algorithms with a minimum energy requirement for a jet, the minimal half opening angle of jet cone $\mathcal{R}$ can be used. The anti- $k_{T}$ [9] or SISCone [10] can serve as examples of such cone-like jet algorithms. For the inclusive clustering algorithms, often used in $e^{ \pm} p$ and $p p$ jet studies, or for the cone-like algorithms [9, 10] with fixed $\mathcal{R}$ parameter, the number of hard jets is defined with a cut on jet (transverse) momentum or jet (transverse) energy [7].

The quantities of interest measured in $e^{+} e^{-}$jet studies [2 5] are $\mathcal{N}$-jet rates and the distribution of transition parameters $D_{i, i+1}$. The $\mathcal{N}$-jet rates are defined as a ratio of production cross section of events with $\mathcal{N}$ jets at some $y$ value to the total hadronic cross section. The transition parameters $D_{i, i+1}$ are defined as values of $y$ for which the event changes from $i$ to $i+1$ jets.

In the $e^{ \pm} p$ jet studies the quantities of interest are the multi-jet cross sections in bins of transverse momentum (energy) of jets and photon virtuality [11,12] and inclusive jet cross sections [13]. Similarly, in the $p p$ jet studies the multi-jet differential cross sections are measured in bins of transverse momentum of leading jets [14].

\section{$3 \quad$ Event classes and relation to other jet observables}

\subsection{Event classes}

An application of jet algorithm with a fixed set of cut parameters $\left\{y_{1}<y_{2}<\cdots<y_{n}\right\}$ results in set $C=\left\{r_{1} r_{2} \ldots r_{n}\right\}$ of natural numbers for each event, where $r_{i}$ corresponds to number of jets obtained with cut parameter $y_{i}$. As events with same $C$ have the same topology, $C$ is used to group events in classes. To limit the number of possible classes, events with number of jets $\mathcal{N}<\mathcal{N}_{\text {min }}$ are treated as events with $\mathcal{N}_{\text {min }}$ jets and events with number of jets $\mathcal{N}>\mathcal{N}_{\text {max }}$ as events with $\mathcal{N}_{\text {max }}$ jets. In the general case the total 
number of classes, $K$, is 1

$$
K=\left(\mathcal{N}_{\max }-\mathcal{N}_{\min }+1\right)^{n}
$$

For the exclusive $k_{T}$ algorithm in $e^{+} e^{-}$collisions, the number of jets rises monotonically with the decreasing cut parameter $y$ and the number of classes is smaller 2 :

$$
K=\frac{\left(n+\mathcal{N}_{\max }-\mathcal{N}_{\min }\right) !}{n !\left(\mathcal{N}_{\max }-\mathcal{N}_{\min }\right) !}
$$

An example of event classes distribution in $e^{+} e^{-}$collisions is given in Fig. 1 ,

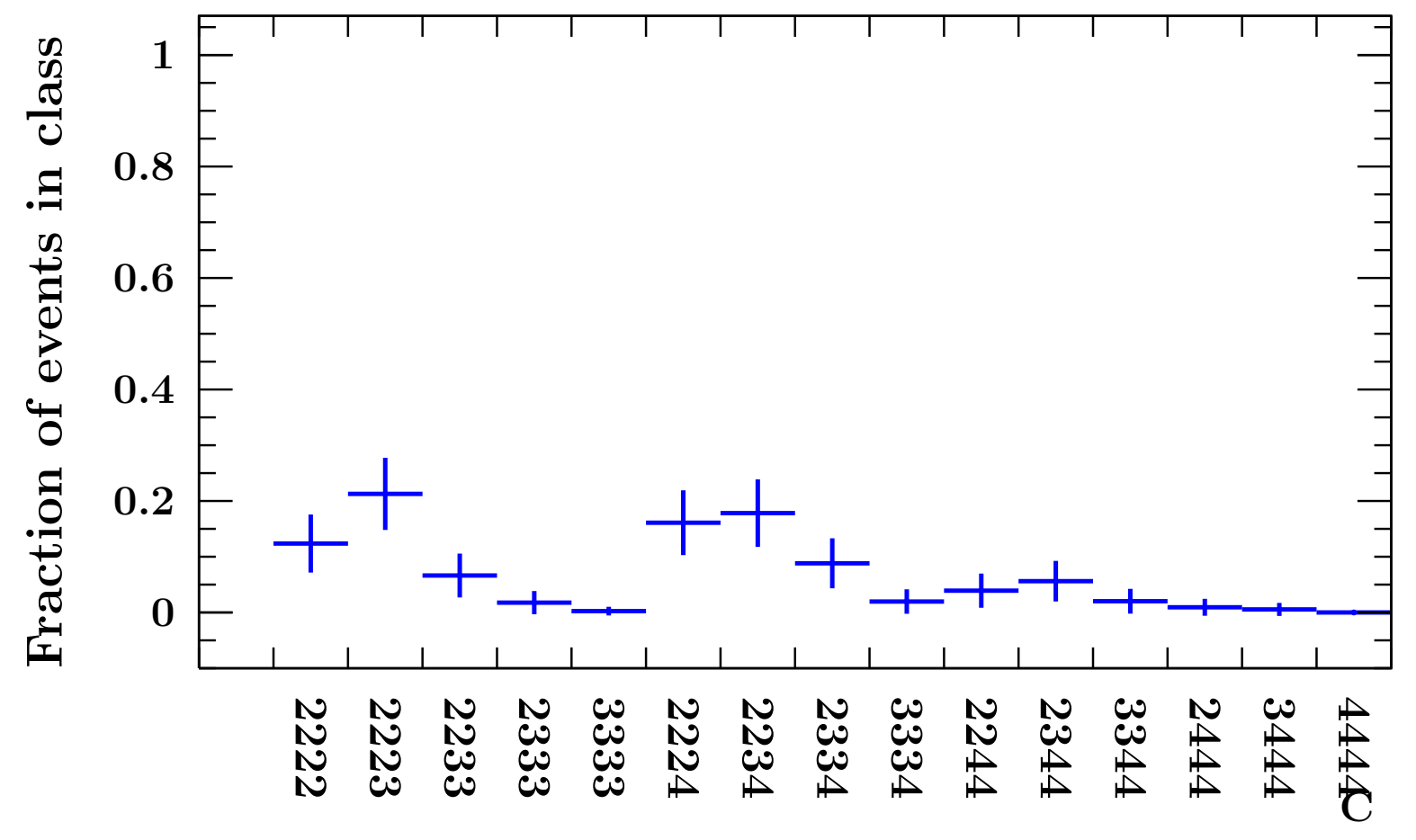

Figure 1: Fractions of event classes obtained with the exclusive $k_{T}$ algorithm at particle level from $e^{+} e^{-}$sample simulated with SHERPA 2.2.1 using $\alpha_{s}\left(M_{Z}\right)=0.12$. The vertical error bars show the square roots of the covariance matrix diagonal elements multiplied by factor 50 for better visibility.

The sample contains 100000 events simulated with the SHERPA 2.2.1 MC generator [15] at centre-of-mass-energy $91 \mathrm{GeV}$ (see Listing 1 for details). The exclusive $k_{T}$ algorithm as implemented in FastJet 3.1.3 [16] is applied at particle level. The used cut parameters

\footnotetext{
${ }^{1}$ For each $y_{i}$ the number of jets can be between $\mathcal{N}_{\text {min }}$ and $\mathcal{N}_{\text {max }}$. Hence, $K$ is a number of combinations of $\mathcal{N}_{\max }-\mathcal{N}_{\min }+1$ distinct values by $n$ with repetitions.

${ }^{2}$ Let $u$ s add $y_{0}$ to the set of cut parameters and set $r_{0}=N_{\max }-r_{1}$. With this addition, every $r_{i} \geq 0$ and their sum is $\mathcal{N}_{\max }-\mathcal{N}_{\min }$. Hence, $K$ is a number of weak compositions of $\mathcal{N}_{\max }-\mathcal{N}_{\min }$ by $n+1$.
} 
are subset of these in Ref. [3]

$$
\{0.1000,0.0215,0.0046,0.0010\}
$$

and $\mathcal{N}_{\text {min }}=2, \mathcal{N}_{\text {max }}=4$.

Similarly, for the anti- $k_{T}$ algorithm [9] in $p p$ collisions, the number of jets rises monotonically with the decreasing $p_{T}$ cut, which acts as the cut parameter in this case. An example of event classes distribution in $p p$ collisions is given in Fig. 2. The sample contains

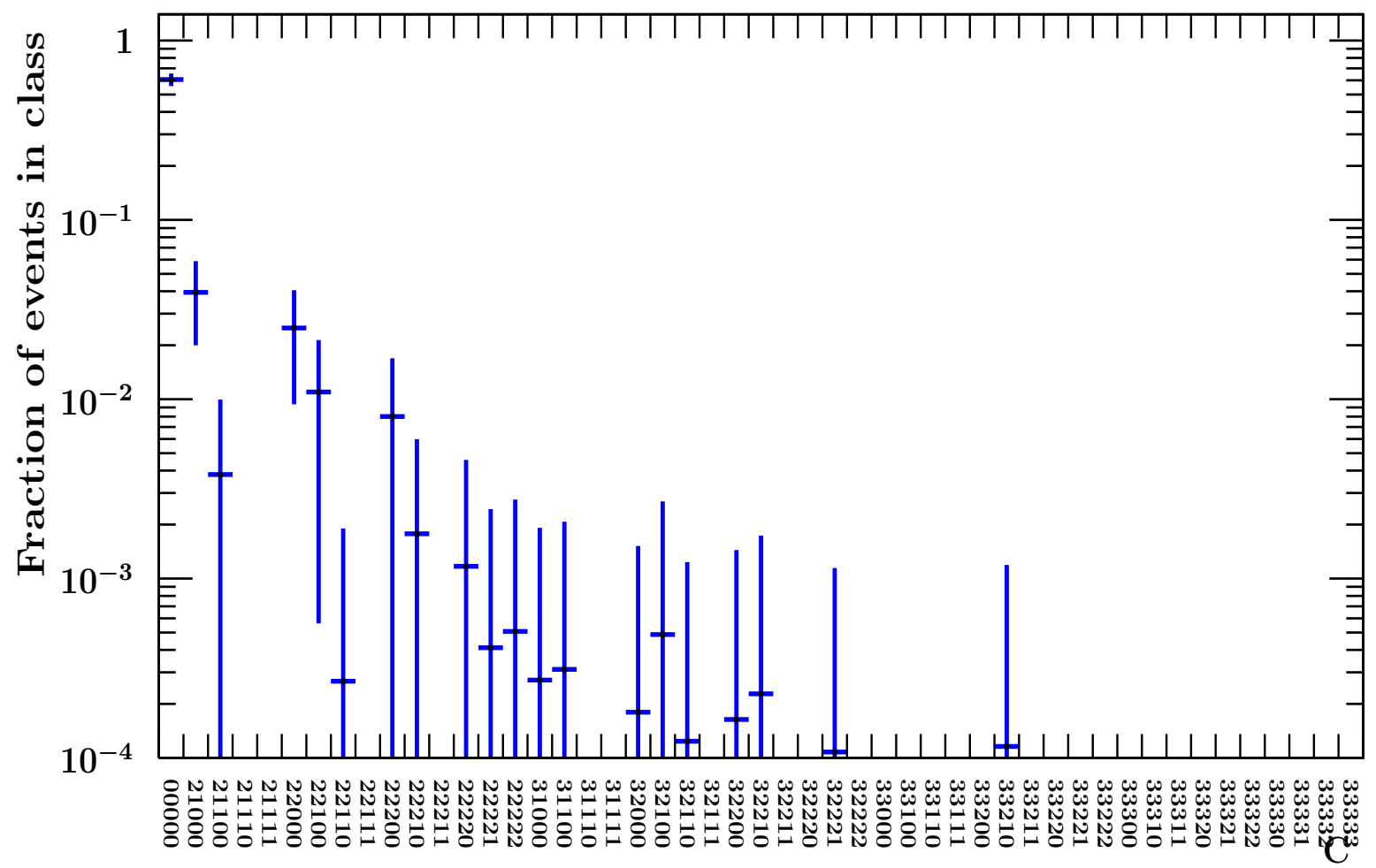

Figure 2: Distribution of event classes obtained with the anti- $k_{T}$ algorithm at particle level from pp sample simulated with SHERPA 2.2.1. The vertical error bars show the square roots of the covariance matrix diagonal elements multiplied by factor 10 for better visibility. Bin labelled with 00000 contains events rejected with selection cuts.

250000 events simulated with the SHERPA 2.2.1 MC generator [15] at centre-of-massenergy $7 \mathrm{TeV}$ (see Listing 2 for details). The anti- $k_{T}$ jet algorithm from FastJet 3.1.3 [16] with the radius parameter $\mathcal{R}=0.4$ is used at particle level. Similarly to Ref. [14], only jets with transverse momenta $p_{T}>60 \mathrm{GeV}$ and rapidity $|y|<2.8$ are considered. The leading jet is further required to have $p_{T}>80 \mathrm{GeV}$. The used $p_{T}$ cuts are

$$
\{60,80,110,160,210\} \mathrm{GeV}
$$


and $\mathcal{N}_{\text {min }}=0, \mathcal{N}_{\text {max }}=3$.

The jet radius can act as a cut parameter as well. However, the number of jets does not change monotonically with the change of radius parameter and the total number of classes follows Eq. 2. An example of usage of jet radius as a cut parameter can be found in the jet studies in deep-inelastic scattering in $e^{ \pm} p$ collisions [13. An example of event classes distribution for this case is given in Fig. 3. The sample contains 500000 weighted events

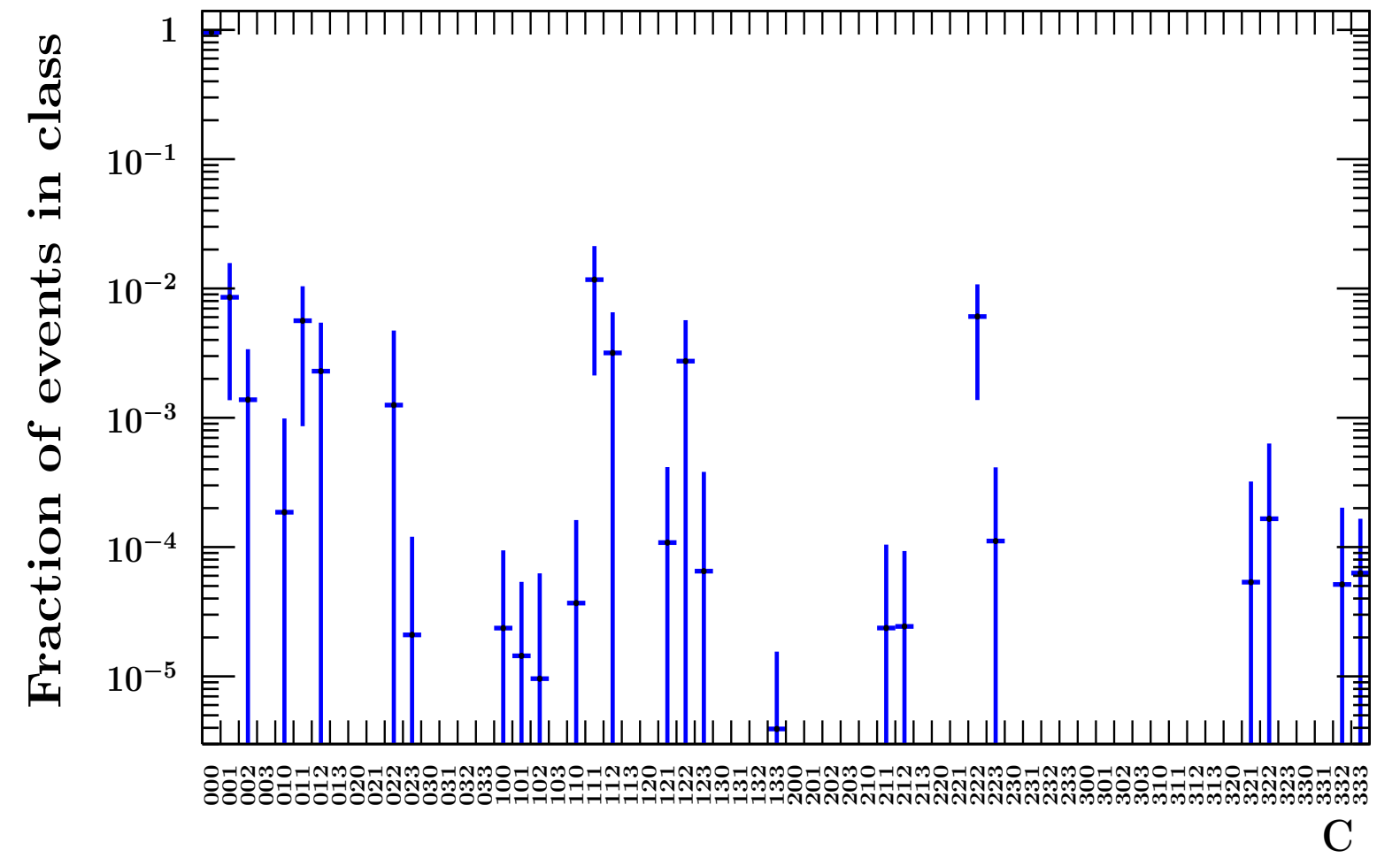

Figure 3: Distribution of event classes obtained with the $k_{T}$ algorithm at particle level from $e^{-} p$ sample simulated with SHERPA 2.2.1. The vertical error bars show the square roots of the covariance matrix diagonal elements multiplied by factor 5 for better visibility. Bin labelled with 000 contains events rejected with selection cuts.

simulated with the SHERPA 2.2.1 MC generator [15] at centre-of-mass-energy $318 \mathrm{GeV}$. The $k_{T}$ jet algorithm is used at particle level in the Breit frame. The implementation from FastJet 3.1.3 [16] is modified to calculate distances between jets with transverse energy as in Ref. [13]. The selected kinematic range is $125<Q^{2}<5000 \mathrm{GeV}^{2}$ and $\left|\cos \gamma_{h}\right|<0.65$, where $Q^{2}=-|\vec{q}|^{2}$ with $\vec{q}$ standing for the four momentum of the exchanged boson and $\gamma_{h}$ is equivalent to the polar angle of the scattered quark in the leading-order quarkparton model [17]. Only jets with transverse energy $E_{T}^{B}>8 \mathrm{GeV}$ and pseudorapidity $-2<\eta^{B}<1.5$ in Breit frame and $E_{T}^{L}>2.5 \mathrm{GeV}$ in laboratory frame are considered. Events contained jets with pseudorapidity $\eta^{L}<-2$ in the laboratory frame are removed. 
The used jet radius parameters are

$$
\{0.5,0.7,1.0\}
$$

and $\mathcal{N}_{\text {min }}=0, \mathcal{N}_{\text {max }}=3$

\subsection{Relation to jet rates in $e^{+} e^{-}$collisions}

The fraction of classes in $e^{+} e^{-}$collisions can be related to $\mathcal{N}$-jet rates and transition parameters. The fraction of events of a given class is denoted with $\kappa_{r_{1} r_{2} \ldots r_{n}}$ or $\kappa_{i=1 \ldots K}$.

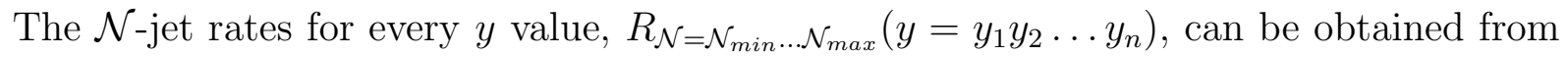
the fraction of events in classes with

$$
R_{\mathcal{N}}\left(y_{i}\right)=\sum_{\left\{r_{i}=N\right\}} \kappa_{1 \ldots K}
$$

For instance, for the chosen set of cut parameters from Eq. 4 for the exclusive $k_{T}$ algorithm

$$
R_{3}\left(y_{2}\right)=\kappa_{2333}+\kappa_{3333}+\kappa_{3334}+\kappa_{3344}
$$

In a general case for all jet rates this can be expressed as a linear transformation from fraction of event classes:

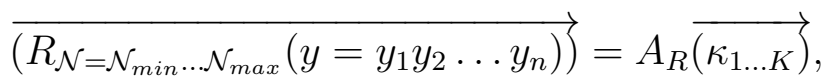

where the matrix $A_{R}$ is constructed to sum all the contributions from all classes to the particular $R_{\mathcal{N}=\mathcal{N}_{\text {min }} \ldots \mathcal{N}_{\text {max }}}\left(y=y_{1} y_{2} \ldots y_{n}\right)$. This relation by construct delivers results identical to a simple jet counting. In the particular case of the chosen set of cut parameters

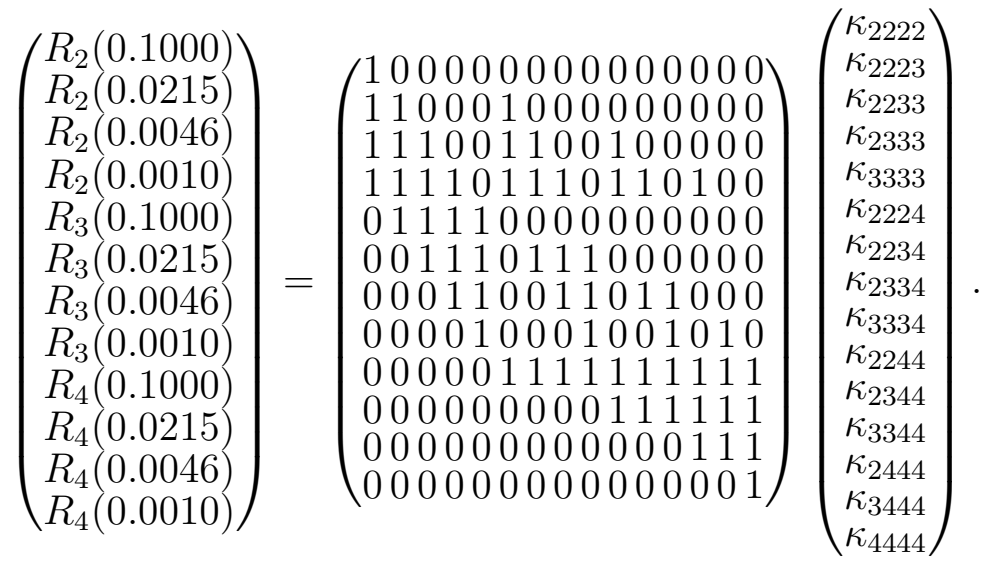

The jet rates obtained with Eq. 10 from the distribution in Fig. 1 are shown in Fig. 4 . 


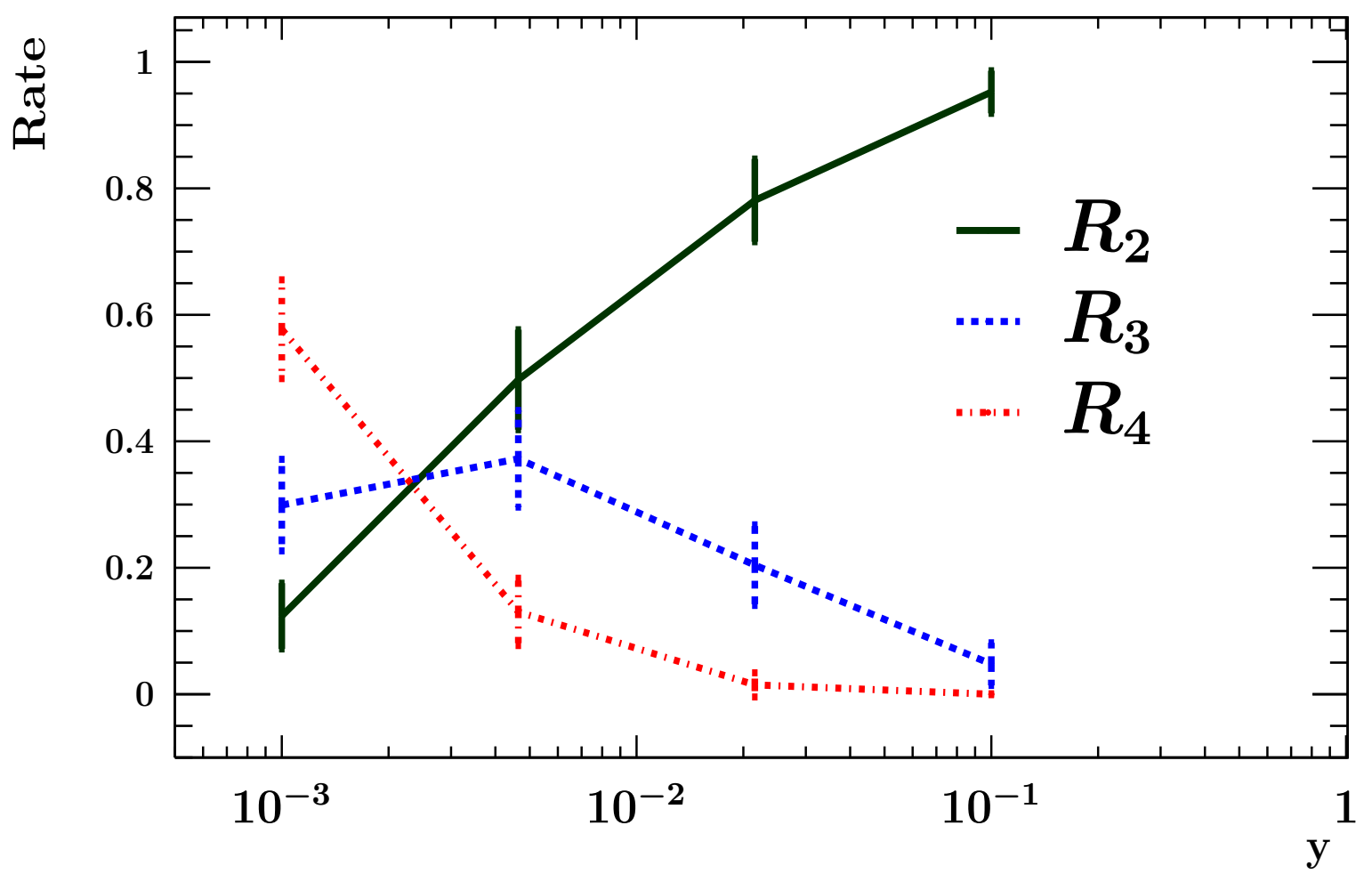

Figure 4: Jet rates obtained with the exclusive $k_{T}$ algorithm at particle level from $e^{+} e^{-}$sample simulated with SHERPA 2.2.1 using $\alpha_{s}\left(M_{Z}\right)=0.12$. The vertical error bars show the square roots of the covariance matrix diagonal elements. For better visibility the size of error bars is scaled by factor 50 and individual points are connected with straight lines.

\subsection{Relation to differential jet rates in $e^{+} e^{-}$collisions}

The fraction of events with transition parameters in bins $\left[y_{j}: y_{j+1}\right]$ can be obtained similarly to the jet rates:

$$
\overrightarrow{\left(D_{i, i+1}\left[y_{j}: y_{j+1}\right], j<n-1, i=\mathcal{N}_{\min } \ldots \mathcal{N}_{\max }-1\right)}=A_{D} \overrightarrow{\left(\kappa_{1 \ldots K}\right)} .
$$

The differential-jet-multiplicity distributions [18] can be obtained from $D_{i, i+1}\left[y_{j}: y_{j+1}\right]$ distributions with a division by corresponding bin width $y_{j}-y_{j+1}$. In the particular case 
of the chosen set of cut parameters

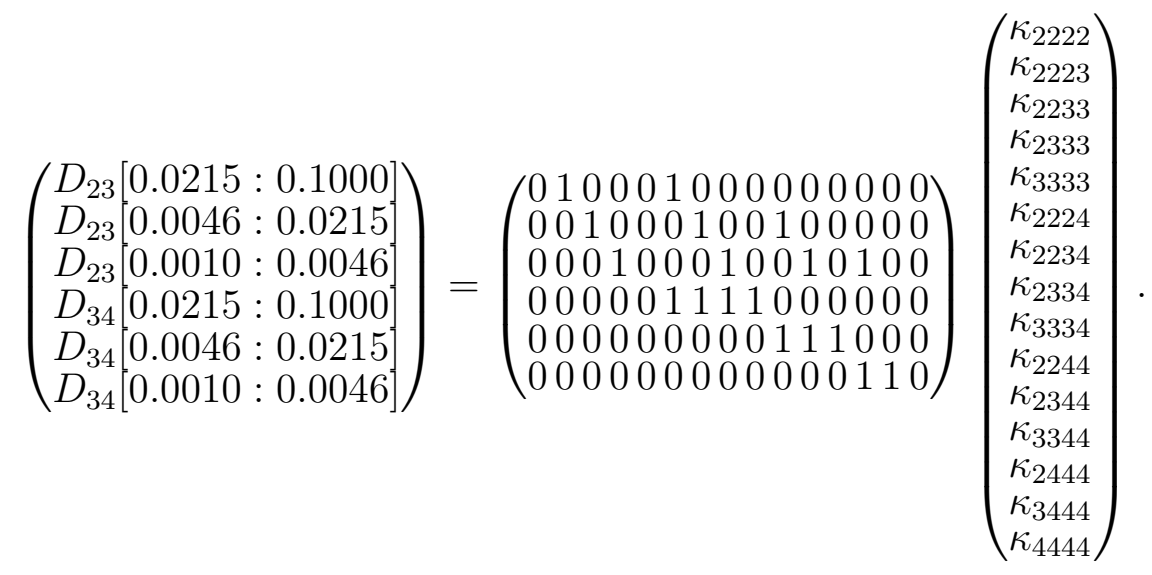

\subsection{Relation to multijet cross sections in $p p$ collisions}

The distribution of classes in $p p$ collisions, obtained above, can be related to multijet cross sections. Similarly to the $e^{+} e^{-}$case, a matrix $A_{L J}$ can be constructed to give

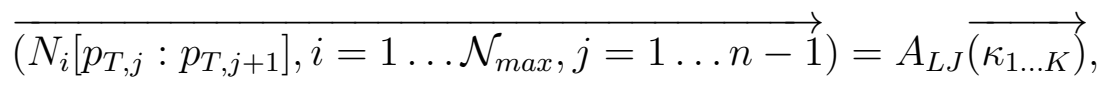

where $N_{i}\left[p_{T, j}: p_{T, j+1}\right]$ stands for the number of events with $i$-th leading jet in bin $\left[p_{T, j}\right.$ : $\left.p_{T, j+1}\right]$. A division of numbers $N_{i}\left[p_{T, j}: p_{T, j+1}\right]$ by the bin widths $p_{T, j+1}-p_{T, j}$ an luminosity of the simulated sample will deliver jet cross sections in corresponding bins. In the particular case of the chosen set of $p_{T}$ cuts and additional requirements [14] we have:

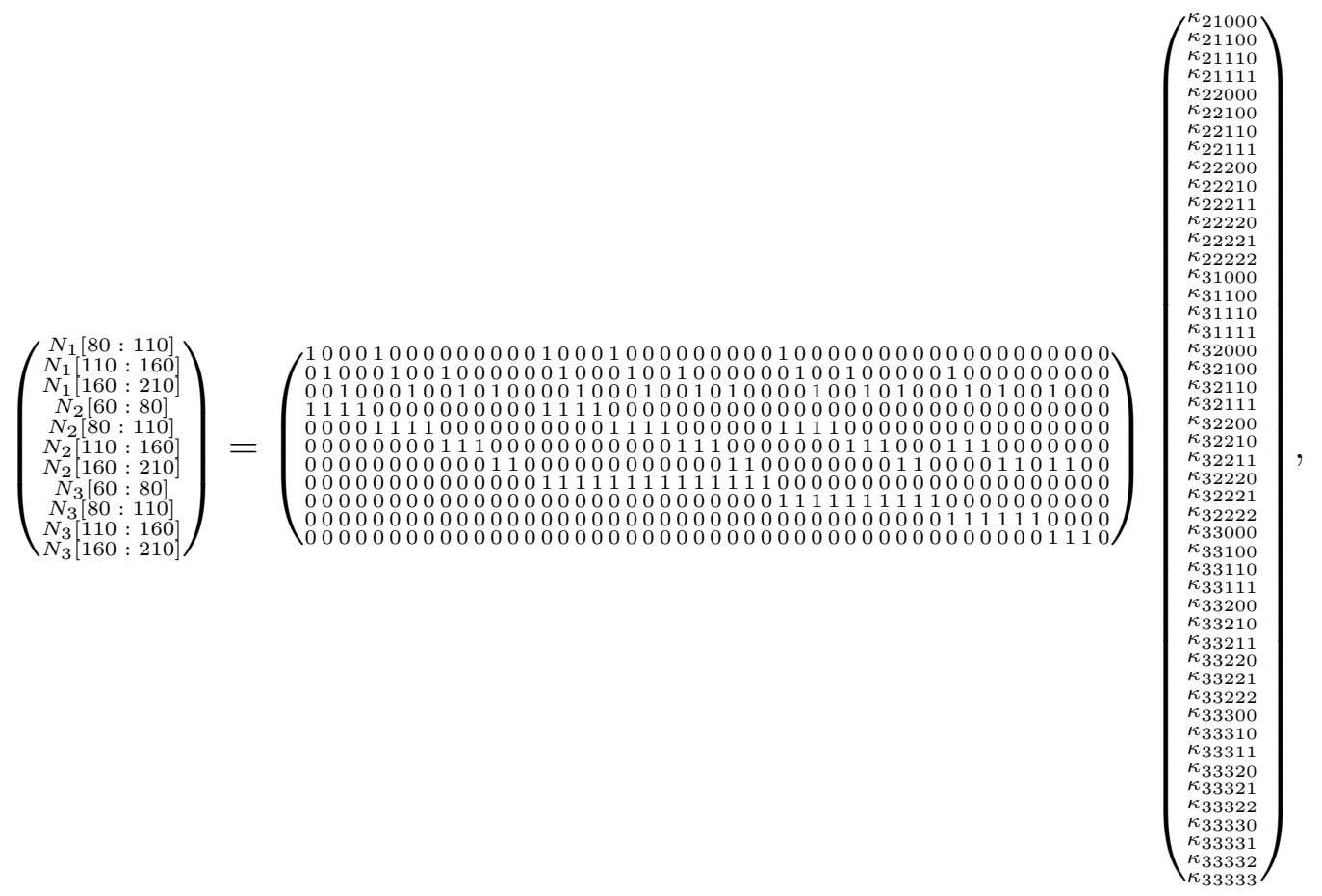


where the boundaries of $p_{T}$ bin are given in $\mathrm{GeV}$. The multijet cross sections obtained in this way from the distribution in Fig. 2 are shown in Fig. 5 .

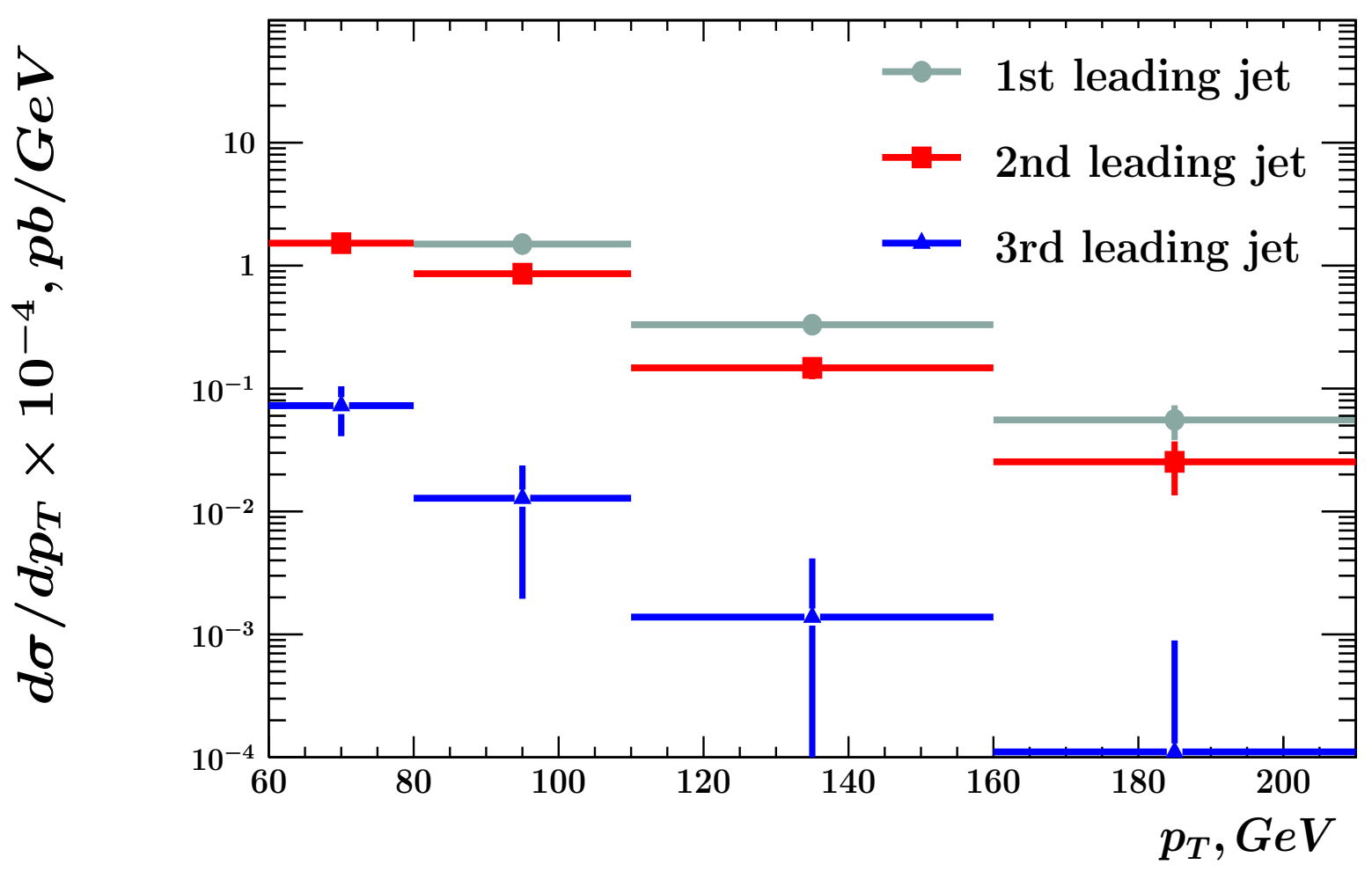

Figure 5: Multijet cross sections obtained with the anti- $k_{T}$ algorithm at particle level from pp sample simulated with SHERPA 2.2.1. The vertical error bars show the square roots of the covariance matrix diagonal elements. For better visibility the size of error bars is scaled by factor 10.

\subsection{Relation to inclusive jet cross sections in $e^{ \pm} p$ collisions}

The distribution of classes in $e^{-} p$ collisions, obtained above, can be related to inclusive jet cross sections. The corresponding matrix $A_{I J}$ can be constructed to give

$$
\left.\overrightarrow{\left(N\left(\mathcal{R}=\mathcal{R}_{i}\right), i=1 \ldots n\right.}\right)=A_{I J} \overrightarrow{\left(\kappa_{1 \ldots K}\right)},
$$

where $N\left(\mathcal{R}=\mathcal{R}_{i}\right)$ stands for the number of inclusive jets obtained with radius parameter $\mathcal{R}_{i}$. Unlike $A_{L J}$, the $A_{I J}$ matrix contains coefficients greater than one, i.e. 2 for events with two jets and 3 for events with three jets. In the particular case of chosen radius 
parameters

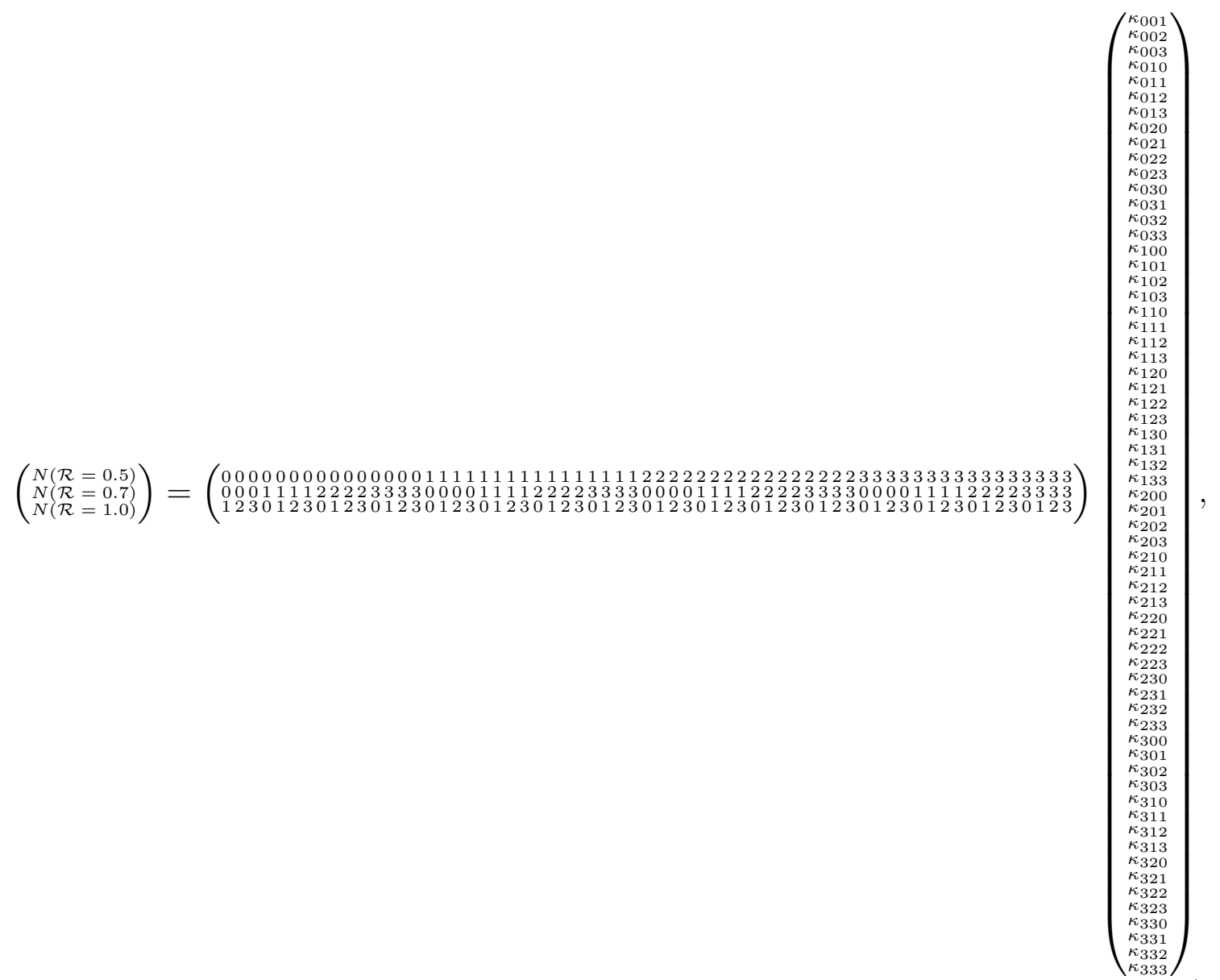

(16)

A division of the $N\left(\mathcal{R}=\mathcal{R}_{i}\right)$ numbers by the luminosity of the simulated sample delivers inclusive jet cross sections shown in Fig. 6 .

\section{Statistical correlations}

In this section different methods to calculate the statistical correlations between jet observables are described. As every experimental measurement has to be corrected for instrumental (detector) effects, particular interest has the propagation of these corrections within every method.

\subsection{Classes-bases method}

In the classes-based method every event is assigned to a class uniquely and the number of events in every class (e.g. at detector level in data or at particle level in MC simulated 


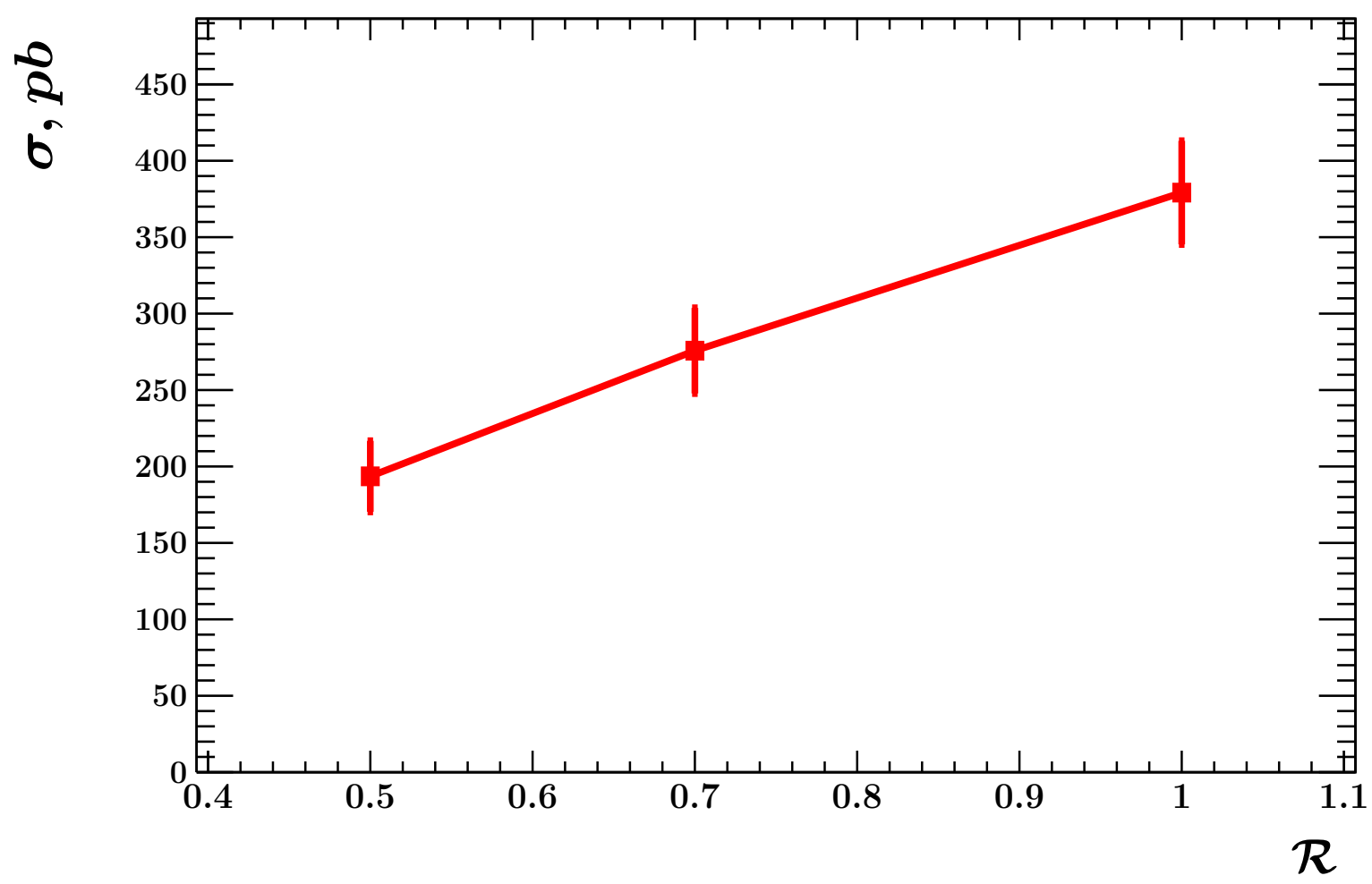

Figure 6: Inclusive jet cross sections obtained with the $k_{T}$ algorithm at particle level from $e^{-} p$ sample simulated with SHERPA 2.2.1. The vertical error bars show the square roots of the covariance matrix diagonal elements. For better visibility the size of error bars is scaled by factor 5 and individual points are connected with straight lines.

events) are statistically independent. Hereby, in the discussed cases of $p p$ and $e^{ \pm} p$ collisions, the number of events in every class, $\kappa_{i}$, the corresponding covariance matrices are diagonal

$$
V_{i j}^{\kappa}=\delta_{i j} \kappa_{i}
$$

The covariance matrix for multijet cross sections, $V^{L J}$, can be obtained from the $V^{\kappa}$ using the matrix from Eq. 14:

$$
V^{L J}=A_{L J} V^{\kappa} A_{L J}^{T}
$$

The covariance matrix for inclusive jet cross sections, $V^{I J}$, can be obtained from the $V^{\kappa}$ using the matrix from Eq. 16:

$$
V^{I J}=A_{I J} V^{\kappa} A_{I J}^{T} .
$$

The numerical values for corresponding correlation matrices are given in Tab. 1 and Tab. 2.

In the discussed case of $e^{+} e^{-}$collisions, it is more practical to use the fraction of events in every class $\kappa_{i}$. It follows a multinomial distribution with the corresponding covariance 


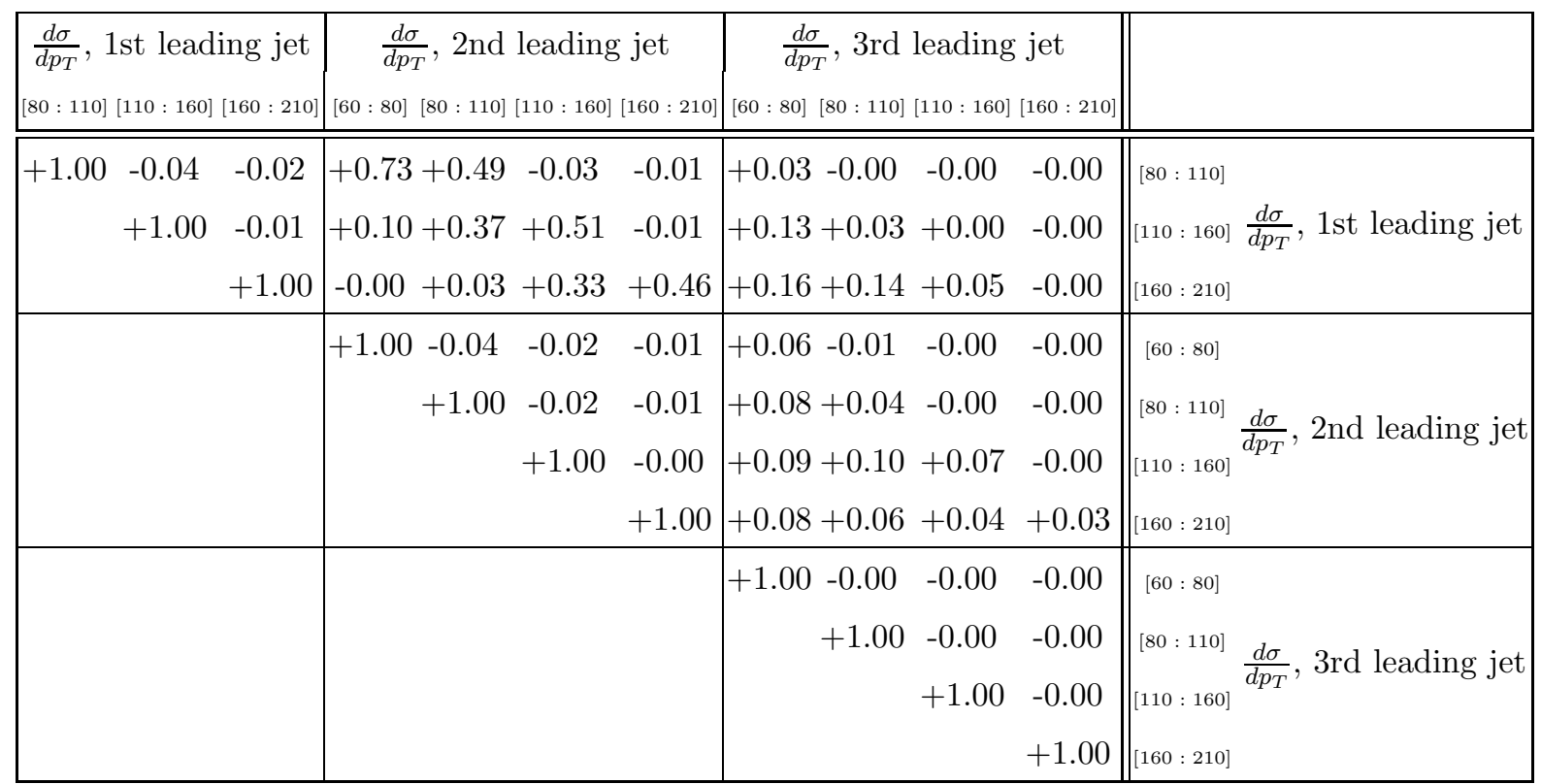

Table 1: The correlation matrix of multijet cross sections in pp collisions in bins of jet transverse momenta (given in brackets) calculated with classes-based method.

\begin{tabular}{|ccr||c|}
\hline$\sigma(\mathcal{R}=0.5) \sigma(\mathcal{R}=0.7) \sigma(\mathcal{R}=1.0)$ & \\
\hline \hline$+1.00 \quad+0.87$ & +0.76 & $\sigma(\mathcal{R}=0.5)$ \\
& +1.00 & +0.89 & $\sigma(\mathcal{R}=0.7)$ \\
& & +1.00 & $\sigma(\mathcal{R}=1.0)$ \\
\hline
\end{tabular}

Table 2: The correlation matrix of inclusive jet cross sections in $e^{-} p$ collisions obtained with different radius parameter $\mathcal{R}$ calculated with classes-based method.

matrix

$$
V_{i j}^{\kappa}=\kappa_{i}\left(\delta_{i j}-\kappa_{j}\right) .
$$

Therefore, covariance matrices for the jet rates $V^{R}$ and transition parameters $V^{D}$ can be obtained from the $V^{\kappa}$ using the matrices from Eq. 10 and Eq. 12,

$$
V^{R}=A_{R} V^{\kappa} A_{R}^{T}, V^{D}=A_{D} V^{\kappa} A_{D}^{T}
$$

The remaining part of this section will be dedicated to descriptions and comparisons to other methods.

\subsection{Direct counting method}

In the recent studies of jet production in $e^{ \pm} p$ collisions [19] the problem of the estimation of statistical correlations between measured jet observables is addressed. Namely, the 
direct counting method [19] is used to estimate correlations between the measured jet cross sections in bins of transverse energy. The method implies counting of the events which contribute to different bins assuming Poisson distribution for the number of events contributing in different ways. The obtained covariance matrices should be corrected for detector effects. In the recent measurements of multijet jet production in $e^{ \pm} p$ collisions [12, 20] the covariance matrices at particle level are obtained with regularised unfolding method (see Refs. [21,22 for details of implementation). The procedure is straightforward and well established, but depends on the regularisation conditions and can be problematic in case of singular covariance matrix at detector level (e.g. because of small number of data events).

\subsection{Sampling method}

In the studies of jet production in $e^{+} e^{-}$collisions at LEP [3, 5], PETRA [2] and TRISTAN [4] the correlations between measured jet rates were estimated from the sampling of MC simulated events. By choosing large number of events out of the set of all MC simulated events, $N_{\text {subsamples }}$ are built. Then the jet rates are measured at particle level in every subsample and the covariance matrix is estimated as

$$
V_{i j}^{R}=\frac{\sum_{k=1 \ldots N_{\text {subsamples }}}\left(R_{i, k}-\bar{R}_{i}\right)\left(R_{j, k}-\bar{R}_{j}\right)}{N_{\text {subsamples }}-1},
$$

where $k$ enumerates measurements obtained in the $k$-th subsample, $i$ and $j$ indexes correspond to Eq. 10 and bar denotes the mean of quantity over the subsamples. As this method was often used in the studies of jet production in $e^{+} e^{-}$collisions, it is compared to the classes-based method using the relations to jet rates for the exclusive $k_{T}$ algorithm from Sec. 3.2 , i.e. with the same setup as used to produce jet rates in Fig 4 .

\subsection{Comparison of classes-based and sampling methods}

In this study the sampling method is applied to the $e^{+} e^{-}$events simulated with the SHERPA 2.2.1 MC program [15] and clustered with the exclusive $k_{T}$ jet algorithm. In total 1000 subsamples with 100 events each are generated. The used steering card is shown in Listing 1. The correlation matrix, $W_{i j}^{R}=V_{i j}^{R} / \sqrt{V_{i i}^{R} V_{j j}^{R}}$, calculated with the sampling method is given in Tab. 3.

The confidence intervals of the obtained correlation coefficients $\rho=W_{i j}^{R}$ can be calculated using Fisher's $z$-transformation [23]. According to Ref. [23] the variable $z=$ $\operatorname{arctanh}(\rho)$ is distributed normally with standard deviation of $1 / \sqrt{N_{\text {subsamples }}-3}$, hereby, for $N_{\text {subsamples }}=1000$ the $68 \%$ confidence intervals are $[-0.022,0.042]$ for $\rho=0.010$; 


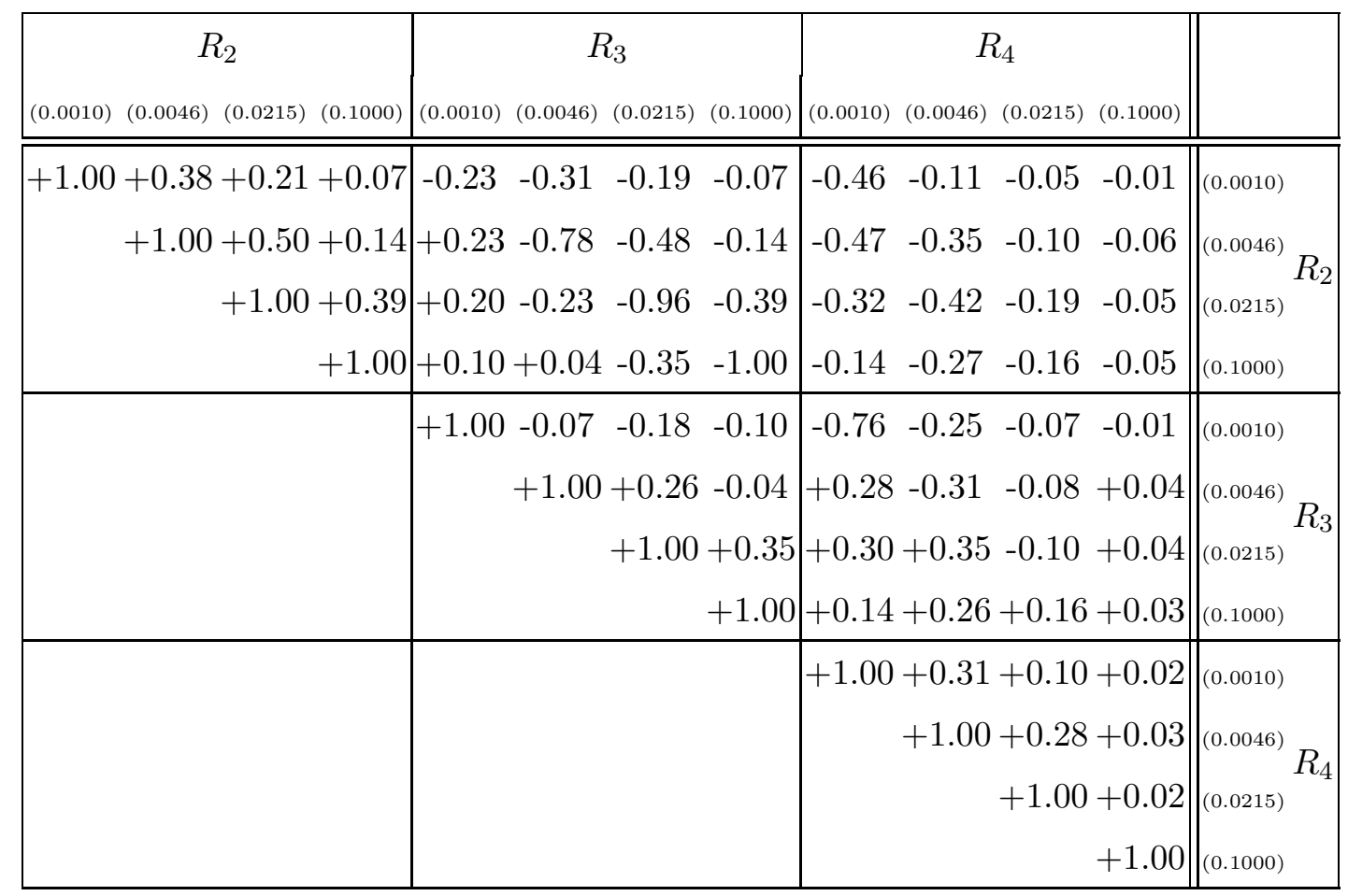

Table 3: The correlation matrix for $e^{+} e^{-}$jet rates $R_{2}, R_{3}$ and $R_{4}$ as functions of cut parameter (given in brackets) calculated with sampling method. The sample is simulated with $\alpha_{s}\left(M_{Z}\right)=0.12$.

$[0.069,0.131]$ for $\rho=0.100 ;[0.579,0.620]$ for $\rho=0.600 ;[0.894,0.906]$ for $\rho=0.900$ and $[0.989,0.991]$ for $\rho=0.990$.

The correlation matrix calculated with Eq. 21 from the same sample is given in Tab. 4. The classes-based method and the sampling method with large number of subsamples give very close results. However, the results obtained with sampling method have sizeable uncertainties, especially for the low values of correlation coefficients. This property of the sampling method comes from the ignoring addition information of event independence within the subsamples.

The stability of classes-based and sampling methods can be also tested and compared in another way. In perturbative QCD jet rates at every $y$ cut value are smooth functions of $\alpha_{s}\left(M_{Z}\right)$ [24]. The correlation coefficients depend on the jet rates, therefore, in case of proper estimation of correlation coefficients, a smooth dependence of correlation coefficients on the $\alpha_{s}\left(M_{Z}\right)$ is expected. To study dependence of the obtained results on $\alpha_{s}\left(M_{Z}\right)$, the calculations from previous sections are repeated with samples generated using $\alpha_{s}\left(M_{Z}\right)=0.09,0.10,0.11$,

$0.12,0.13,0.14,0.15$. Some of the obtained results are shown in Fig. 7. Smooth dependency of correlation coefficients obtained with classes-based method on $\alpha_{s}\left(M_{Z}\right)$ can serve as a proof of method robustness. For both methods significant dependence on the value 


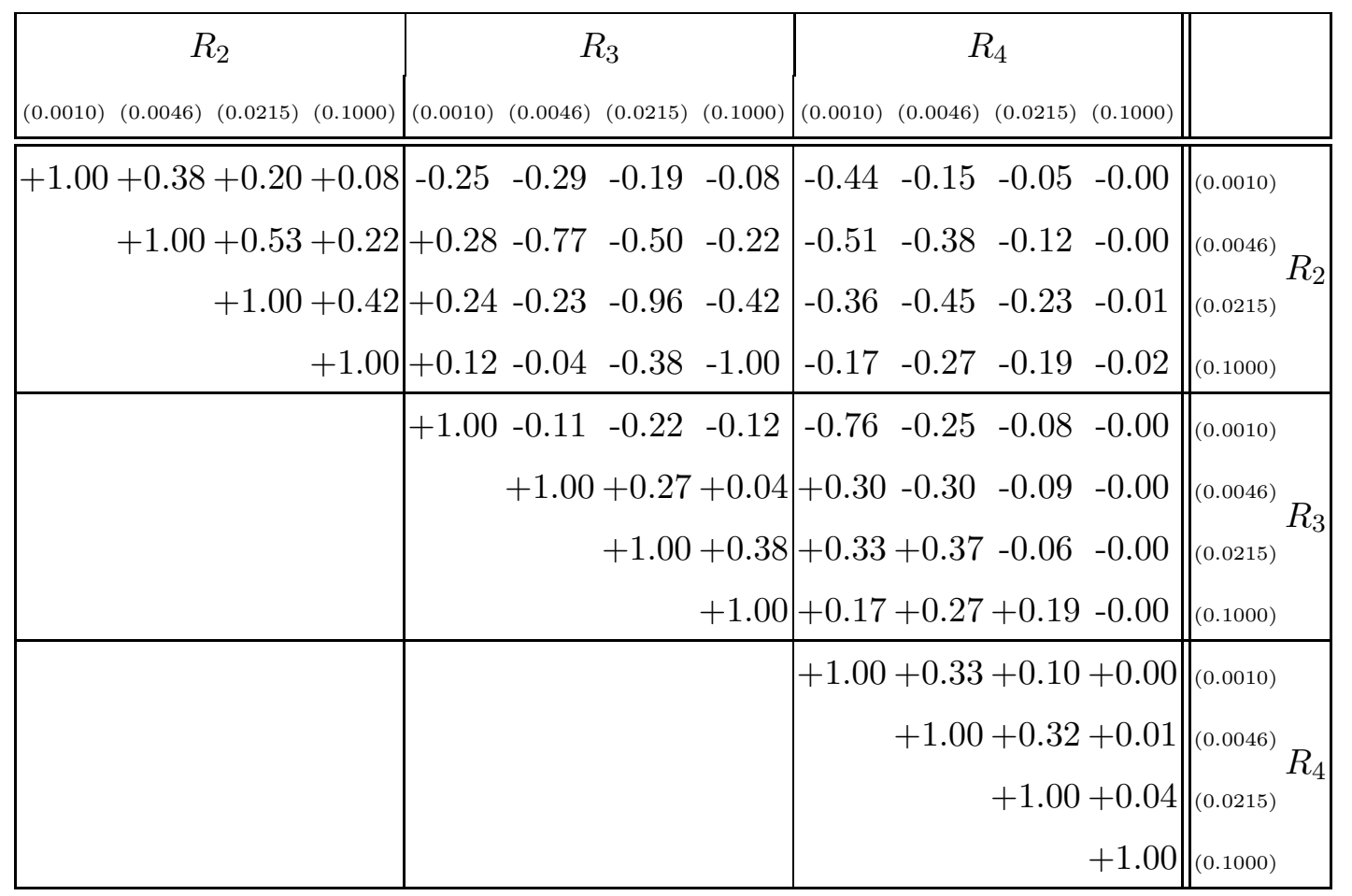

Table 4: The correlation matrix for $e^{+} e^{-}$jet rates $R_{2}, R_{3}$ and $R_{4}$ as functions of cut parameter (given in brackets) calculated with classes-based method. The sample is simulated with $\alpha_{s}\left(M_{Z}\right)=0.12$.

of $\alpha_{s}\left(M_{Z}\right)$ used in the simulation is present. Therefore, to obtain correlation coefficients consistent with data, sampling of data or classes-based method should be used. While for the former option the precision of the obtained correlation coefficients is limited by number of subsamples [23], the classes-based method does not face this problem. In addition, the sampling of data involves complications with corrections of the measured quantities for detector effects. Contrary to that, the correction for detector effects for classes is straightforward, can be done either "bin-by-bin", or with the unfolding procedures and propagated to the final results. 


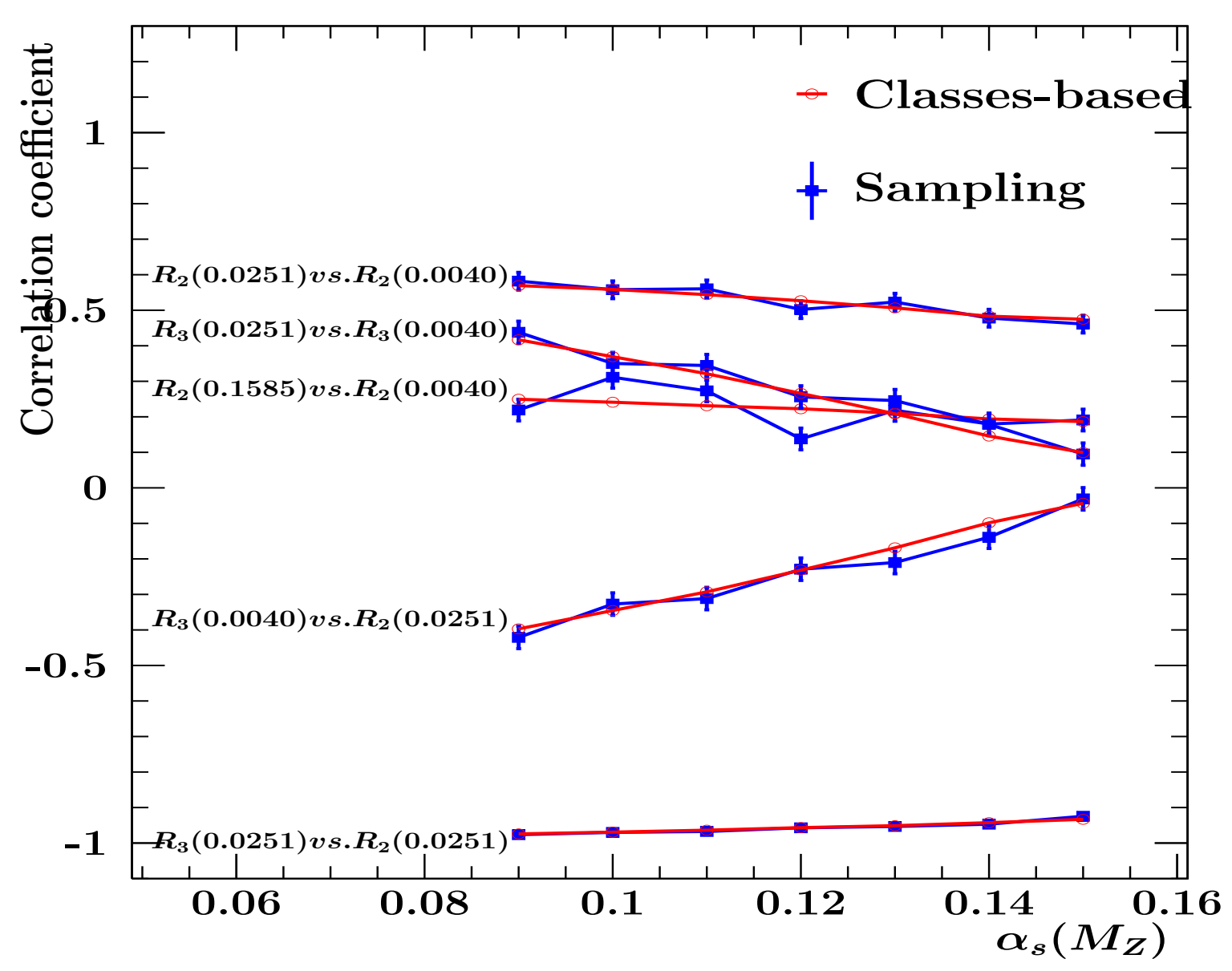

Figure 7: Dependence of selected elements of correlation matrix for jet rates $W^{R}$ calculated with sampling method and with classes-based method on $\alpha_{s}\left(M_{Z}\right)$ used by SHERPA 2.2.1 in simulation. The error bars for the sampling method are the 68\% confidence intervals calculated according to Ref. [23]. For better visibility the individual points are connected with straight lines.

\section{Summary}

A new type of jet observables, classes, is introduced. The classes-based method to calculate correlations between measurements of jet observables is demonstrated. The method provides robust results, does not rely on the MC simulations and has a straightforward, self-consistent procedure for taking into account detector corrections.

\section{Acknowledgements}

I thank Stefan Kluth for his major contribution to the development of this paper. I thank Olaf Behnke and Oleksandr Zenaiev for the discussions on the topic and suggestions for 
the improvements of the manuscript.

\section{Appendix A Example of SHERPA 2.2.1 setup for $e^{+} e^{-}$ collision simulation}

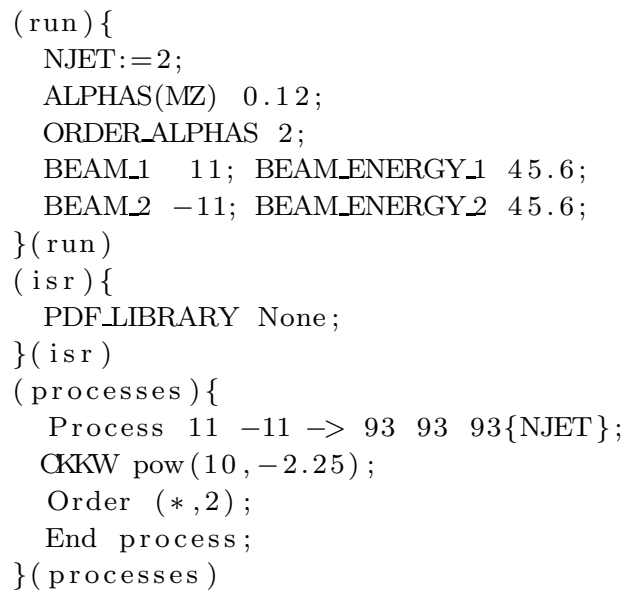

Listing 1: SHERPA 2.2.1 steering card for $e^{+} e^{-}$sample simulated with $\alpha_{s}\left(M_{Z}\right)=$ 0.12 .

\section{Appendix B Example of SHERPA 2.2.1 setup for $p p$ collision simulation}

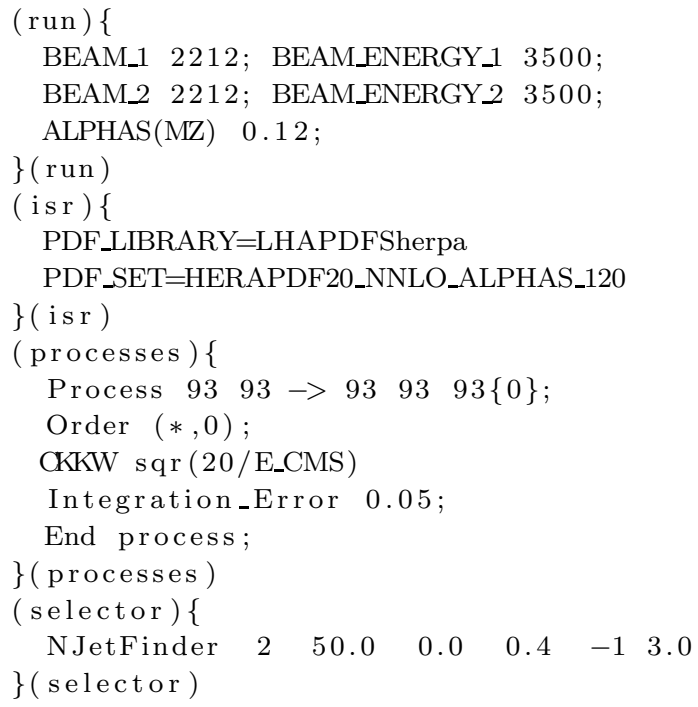

Listing 2: SHERPA2.2.1 steering card for pp sample. 


\section{Appendix C Example of SHERPA 2.2.1 setup for $e^{ \pm} p$ collision simulation}

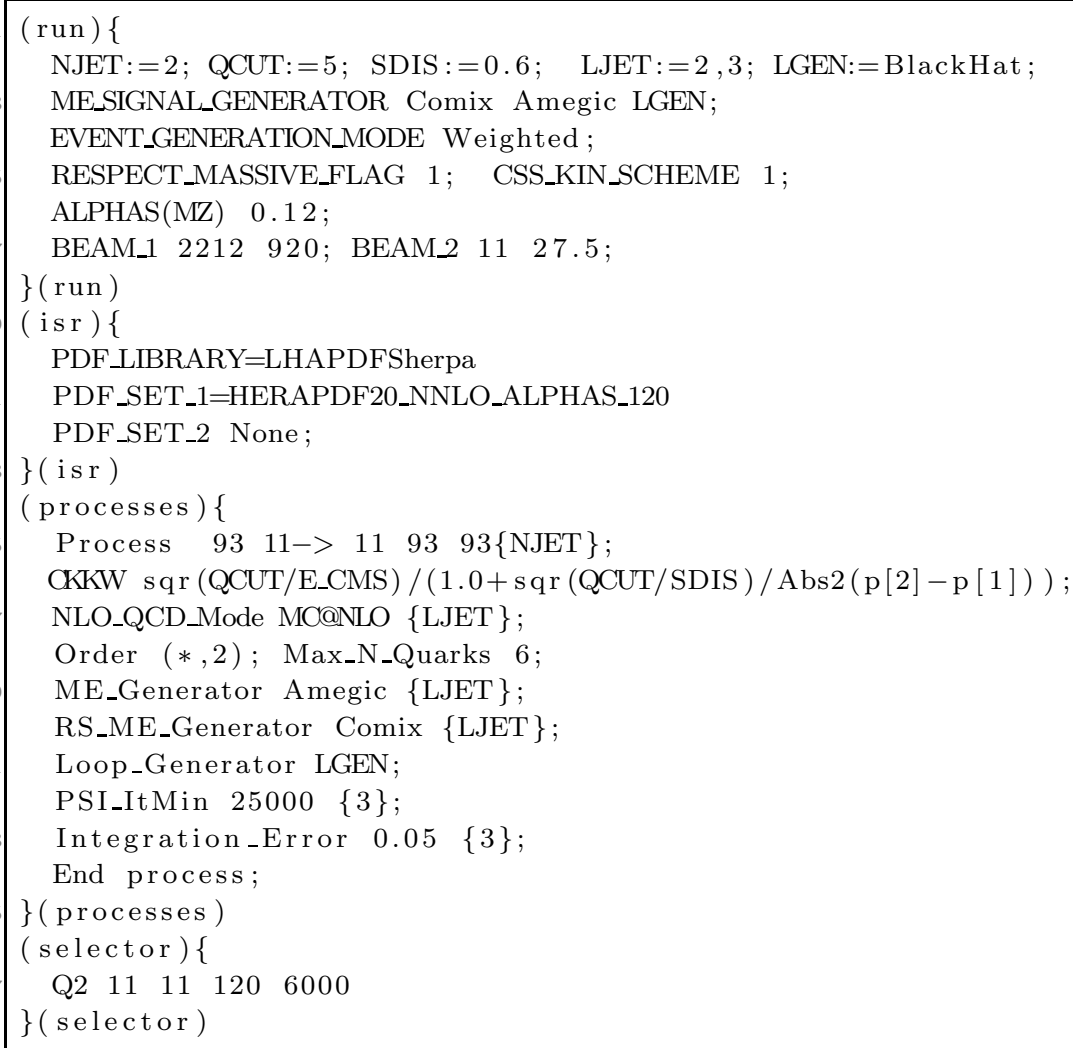

Listing 3: $\quad$ SHERPA 2.2.1 steering card for $e^{+} p$ sample.

\section{References}

[1] G. Dissertori et al., Precise determination of the strong coupling constant at NNLO in QCD from the three-jet rate in electron-positron annihilation at LEP. Phys. Rev. Lett. 104, 072002 (2010), arXiv:0910.4283.

[2] JADE Collaboration, J. Schieck et al., Measurement of the strong coupling $\alpha_{s}$ from the three-jet rate in $e^{+} e^{-}-$annihilation using JADE data.

Eur. Phys. J. C73, 2332 (2013), arXiv:1205.3714.

[3] OPAL Collaboration, G. Abbiendi et al., Determination of $\alpha_{s}$ using OPAL hadronic event shapes at $\sqrt{s}=91-209 \mathrm{GeV}$ and resummed NNLO calculations. Eur. Phys. J. C71, 1733 (2011), arXiv:1101.1470. 
[4] M.E. Zomorrodian, M. Hasheminia and A. Mirjalili, Measurement of strong coupling $\alpha_{s}$ in $e^{+} e^{-}$annihilation using jet rate and event shape. Indian J. Phys. 90, 469 (2016).

[5] ALEPH Collaboration, A. Heister et al., Studies of QCD at $e^{+} e^{-}$centre-of-mass energies between $91 \mathrm{GeV}$ and $209 \mathrm{GeV}$. Eur. Phys. J. C35, 457 (2004).

[6] A. Ali and G. Kramer, Jets and QCD: a historical review of the discovery of the quark and gluon jets and its impact on QCD. Eur. Phys. J. H36, 245 (2011). arXiv:1012.2288.

[7] T. Schörner-Sadenius, Jet physics in electron-proton scattering. Eur. Phys. J. C72, 2060 (2012). [Erratum: Eur. Phys. J.C72,2133(2012)].

[8] S. Catani et al., New clustering algorithm for multi-jet cross-sections in $e^{+} e^{-}$ annihilation. Phys. Lett. B269, 432 (1991).

[9] M. Cacciari, P.G. Salam and G. Soyez, The anti- $k_{T}$ jet clustering algorithm. JHEP 04, 063 (2008), arXiv:0802.1189.

[10] G.P. Salam and G. Soyez, A practical seedless infrared-safe cone jet algorithm. JHEP 05, 086 (2007), arXiv:0704.0292.

[11] ZEUS Collaboration, S. Chekanov et al., Multijet production in neutral current deep inelastic scattering at HERA and determination of $\alpha_{s}$. Eur. Phys. J. C44, 183 (2005), arXiv:hep-ex/0502007.

[12] H1 Collaboration, V. Andreev et al., Measurement of multijet production in $e^{ \pm} p$ collisions at high $Q^{2}$ and determination of the strong coupling $\alpha_{s}$. Eur. Phys. J. C75, 65 (2015), arXiv:1406.4709.

[13] ZEUS Collaboration, S. Chekanov et al., Jet-radius dependence of inclusive-jet cross-sections in deep inelastic scattering at HERA. Phys. Lett. B649, 12 (2007). arXiv: hep-ex/0701039.

[14] ATLAS Collaboration, G. Aad et al., Measurement of multi-jet cross sections in proton-proton collisions at a $7 \mathrm{TeV}$ center-of-mass energy. Eur. Phys. J. C71, 1763 (2011), arXiv:1107.2092.

[15] T. Gleisberg et al., Event generation with SHERPA 1.1. JHEP 02, 007 (2009). arXiv:0811.4622.

[16] M. Cacciari, P.G. Salam and G. Soyez, FastJet user manual. Eur. Phys. J. C72, 1896 (2012), arXiv:1111.6097.

[17] S. Bentvelsen, J. Engelen and P. Kooijman, Reconstruction of $\left(x, Q^{2}\right)$ and extraction of structure functions in neutral current scattering at HERA, Workshop 
on Physics at HERA Hamburg, Germany, October 29-30, 1991, pp. 0023-42. (1992). https://lib-extopc.kek.jp/preprints/PDF/1992/9209/9209099.pdf.

[18] S. Komamiya et al., Determination of $\alpha_{s}$ from a differential jet multiplicity distribution at SLC and PEP. Phys. Rev. Lett. 64, 987 (1990),

[19] D. Lontkovskyi, Measurement of jet production with the ZEUS detector, Ph.D. thesis, Universität Hamburg, 2015.

http://ediss.sub.uni-hamburg.de/volltexte/2016/7801/pdf/Dissertation.pdf.

[20] H1 Collaboration, V. Andreev et al., Measurement of jet production cross sections in deep-inelastic $e^{ \pm} p$ scattering at HERA . (2016). arXiv:1611.03421.

[21] T. Adye, Unfolding algorithms and tests using RooUnfold, Proceedings of PHYSTAT 2011 Workshop, CERN, Geneva, Switzerland 17-20 January 2011, pp. 313-318. CERN, Geneva, CERN (2011). Also in preprint 1105.1160, doi:10.5170/CERN-2011-006.313. arXiv:1105.1160.

[22] S. Schmitt, TUnfold: an algorithm for correcting migration effects in high energy physics. JINST 7, T10003 (2012), arXiv:1205.6201.

[23] R.A. Fisher, On the probable error of a coefficient of correlation deduced from a small sample. Metron 1, 3 (1921). http://digital.library.adelaide.edu.au/dspace/bitstream/2440/15169/1/14.pdf.

[24] S. Weinzierl, Jet algorithms in electron-positron annihilation: Perturbative higher order predictions. Eur. Phys. J. C71, 1565 (2011). arXiv:1011.6247. [Erratum: Eur. Phys. J.C71,1717(2011)]. 